\title{
Desenho do Trabalho, Atitudes de Carreira e Saúde Mental em empresas de Tecnologia da Informação
}

\author{
Emília dos Santos Magnan ${ }^{1, *}$, Marcos Vinicius Amorim", \\ Wagner de Lara Machado ${ }^{1}$, Manoela Ziebell de Oliveira ${ }^{1}$
}

Os ambientes de trabalho se tornam mais complexos na medida em que o dinamismo do mercado aumenta. O objetivo deste estudo foi investigar fatores contemporâneos de carreira, contexto de trabalho e sua relação com desfechos de saúde mental em profissionais de três empresas de Tecnologia da Informação (TI) de Porto Alegre. A amostra foi composta por 220 trabalhadores que responderam a um questionário sociodemográfico e a escalas relacionadas às variáveis de interesse. Os dados foram submetidos a análises de regressão hierárquica e análise de rede. Os resultados mostraram que fatores relacionados à carreira têm maior poder para explicar Engajamento no Trabalho do que Ansiedade, Depressão e Estresse. Este desfecho é mais explicado por fatores do contexto do trabalho. As atitudes da carreira dos profissionais contemporâneos, bem como as características dos desenhos do trabalho se relacionam e impactam em Engajamento e Ansiedade, Depressão e Estresse dos talentos da área de TI. Entende-se que ambos devem ser levados em conta das propostas de gestão de pessoas dessas organizações.

Palavras-chave: trabalho, orientação vocacional, saúde ocupacional.

\section{Work Design, Career Attitudes and Mental Health in Information Technology Companies}

Abstract

Work environments become more complex as the dynamism of the market increases. The aim of this study was to investigate contemporary career factors, work context and their relationship with health outcomes in professionals that work at three Information Technology (IT) companies in Porto Alegre. The sample was composed of 220 workers who answered a sociodemographic questionnaire and scales related to the variables of interest. The data were submitted to hierarchical regression analysis and network analysis. The results showed that career-related factors have greater power to explain Engagement at Work than Anxiety, Depression and Stress. This outcome is more explained by factors of the work context. Career atitudes of contemporary professionals and Work Design are related to Work Engagement and Anxiety, Depression and Stress for IT talents. It is known that both career atitudes and Work design should count for human resources interventions in IT companies.

Keywords: labour, vocational guidance, occupational health

\section{Proyecto de Trabajo, Actitudes de Carrera y Salud Mental en Empresas de Tecnología de la Información}

Resumen

Los entornos de trabajo se vuelven más complejos en la medida en que aumenta el dinamismo del mercado. El objetivo de este estudio fue investigar factores contemporáneo de carrera, y el contexto laboral y su relación con los resultados de salud en profesionales de tres empresas de Tecnología de la Información (TI) en Porto Alegre. La muestra consistió en 220 trabajadores que respondieron un cuestionario sociodemográfico y escalas relacionadas con las variables de interés. Los datos fueron sometidos a análisis de regresión jerárquica y análisis de red. Los resultados mostraron que los factores relacionados con la carrera tienen mayor poder para explicar el compromiso en el trabajo que la ansiedad, la depresión y el estrés. Este desenlace es más explicado por factores del contexto del trabajo. Los factores contemporáneo de carrera, y el contexto laboral están relacionadas y tienen un impacto en el compromiso en el trabajo y la ansiedad, la depresión y el estrés. del talento de TI. Se entiende que ambos deben tenerse en cuenta para las propuestas de gestión de personas de estas organizaciones.

Palabras-clave: trabajo, orientación vocacional, salud ocupacional
* Informações sobre o autor principal:

R. Gonçalves Dias, 810 - Menino Deus- Porto Alegre - RS, 90130-060, Brasil.

E-mail: emiliamagnan@gmail.com 
Compreender a transformação das carreiras e do contexto de trabalho exige uma investigação conjunta e articulada entre diferentes áreas de conhecimento. A relação entre indivíduos e organizações envolve, conforme salientado por Lassance e Sarriera (2009), dimensões pessoais, do emprego, da carreira, organizacionais e sociais. Por isso, nas últimas décadas, o tema atraiu o interesse de diferentes teóricos: da Orientação Profissional, Psicologia Organizacional e do Trabalho, Administração, Sociologia, Psicologia Social, Antropologia, Economia, Ciências Políticas etc. (Baruch, Szűcs, \& Gunz, 2015; Ribeiro \& Zerbini, 2012).

No contexto organizacional, na década de 1990, destacou-se a iminência de uma transformação na forma como se estabelecia a relação entre trabalhador e trabalho, pautada em uma lógica de "ganha-ganha", com vínculos instáveis e de curto prazo (Peiperl \& Baruch, 1997). Desde então, tem-se elaborado e testado teorias que visam a informar como organizar o trabalho a fim de ofertar posições de emprego que sejam atrativas para os talentos e respondam os desafios competitivos das empresas. As teorias de Desenho do Trabalho vêm sendo extensivamente exploradas pela literatura científica com esse objetivo (Parker, Morgeson, \& Johns, 2017). A evolução dos estudos sobre o tema evidenciou a importância dos aspectos motivacionais para a compreensão dos resultados relacionados à produtividade e à qualidade das entregas feitas pelos profissionais (Parker et al., 2017).

Dentre os modelos propostos ao longo dos anos, o de Recursos e Demandas do Trabalho, por exemplo, explica o mecanismo pelo qual o Engajamento no Trabalho (ET) é gerado, bem como sua implicação para a saúde dos trabalhadores (Hakanen, Schaufeli, \& Ahola, 2008). Evidências apontam que elevadas demandas levam a um processo de adoecimento que impede a promoção do engajamento. Já a presença de recursos, como a autonomia, leva ao engajamento no trabalho e minimiza o impacto negativo das demandas (Schaufeli, 2017; Schaufeli \& Taris, 2014). Assim como esse modelo, na última década, foi apresentada uma nova abordagem, mais integrativa, elaborada por Morgeson e Humphrey (2006). Para a realidade brasileira, as intervenções em Desenhos do Trabalho (DT) pautadas nessa referência podem ser compreendidas a partir de quatro categorias e 18 subcategorias (Borges-Andrade, Peixoto, Queiroga, \& Pérez-Nebra, 2019). São elas: (1) características da tarefa (autonomia, variedade, significado, identidade e feedback); (2) características do conhecimento (complexidade, processamento de informações, solução de problemas, variedade de skills e especialização); (3) características sociais (suporte social, interdependência, interações fora da organização e feedback dos outros); (4) características do contexto (ergonômicas, demandas físicas, condições de trabalho, uso de equipamentos).

Embora sejam relevantes para essa área de investigação, os fatores relacionados ao desempenho e possíveis desfechos do comportamento das pessoas nas organizações não são exclusivamente relacionados aos desenhos do trabalho. Por esse motivo, alguns autores já têm feito tentativas de aproximar os estudos sobre as construções individuais das carreiras e sobre os desenhos do trabalho (Hall \& Las Heras, 2009; Ribeiro e Zerbini, 2012). Evidenciando esses esforços, Hall e Las Heras (2009) defenderam que o campo dos desenhos do trabalho e o da carreira não são tão distintos como, inicialmente, pode parecer. Em um texto icônico sobre as diversas forças que estão moldando o futuro das carreiras, Peiperl e Baruch (1997) fizeram a apresentação do conceito "Carreiras Pós-Corporativas". Essas novas formas de inserção no mundo do trabalho são menos limitadas pelas fronteiras organizacionais e contemplam relações de trabalho alternativas como consultorias, emprego temporário, outsourcing, parcerias profissionais, startups e negócios fechados sob demanda. Nelas, evidencia-se a possibilidade de ex1 Movimento que caracterizou as transições de carreira feitas por mulheres com o intuito de poder contemplar de igual forma diferentes aspectos de suas vidas, como os de mãe e trabalhadora (Mainiero \& Sullivan, 2005). trapolar a relação do emprego e do vínculo formal com a empresa. Segundo observações de diferentes autores (Baruch, et al., 2015; Peiperl \& Baruch, 1997), as atitudes dos profissionais neste contexto de maior autonomia e flexibilização foram, por décadas, estudadas e explicadas através de metáforas de carreira. Uma delas é a da Carreira Proteana, que caracteriza um indivíduo orientado por critérios de sucesso subjetivo e que tende a apresentar comportamentos de aprendizagem contínua, autoconsciência, responsabilidade e autonomia (McDonald, Brown, \& Bradley, 2005). Além desta, outra metáfora que merece destaque é a da Carreira Sem-Fronteiras, que apresenta arranjos de independência em relação às estruturas tradicionais de emprego, podendo ser caracterizada tanto por mobilidade física e objetiva, quanto pela psicológica, que diz respeito à percepção que o indivíduo tem sobre seu potencial de mobilidade física (Sullivan \& Baruch, 2009). Por fim, a metáfora da Carreira Caleidoscópica, proposta inicialmente para entender as carreiras femininas e o movimento de "opt-out", destaca que as decisões de carreira são tomadas com base na saliência de diferentes parâmetros que podem se diferenciar ao longo da vida, a saber: Autenticidade, Balanço e Crescimento (Mainiero \& Sullivan, 2005).

Considerando a revisão de literatura apresentada, foi objetivo deste estudo investigar a relação entre atitudes e comportamentos de carreira pós-corporativos (carreira proteana, sem-fronteiras e caleidoscópica), características do trabalho e variáveis de saúde e bem-estar dos profissionais (engajamento no trabalho, depressão, ansiedade e estresse). A fim de elaborar hipóteses que orientaram as análises realizadas, considerou-se que: (1) com o advento de novas formas para planejar o contexto de trabalho e novos comportamentos de carreira, surgem diferentes impactos relacionados ao desenvolvimento de carreira do indivíduo (Baruch \& Vardi 2016); (2) existe uma relação já documentada entre o engajamento no trabalho e a autonomia para a realização das atividades laborais aqueles das carreiras pós-corporativas (Peiperl \& Baruch; 1997), caracterizam-se por maiores níveis de autonomia e autogerenciamento em todos os profissionais (Oliveira \& Gomes, 2014); (4) embora a literatura venha apresentando, com certo entusiasmo, uma nova configuração do mercado de trabalho, as experiências de carreira contemporâneas podem ser negativas e incluir uma série de consequências indesejadas (Baruch \& Vardi, 2016).

\section{Hipóteses}

Com base nas informações apresentadas anteriormente, propôs-se as seguintes hipóteses:

H1. As dimensões das diferentes expressões das carreiras contemporâneas (proteana, sem-fronteiras e caleidoscópica) apresentarão correlação entre si;

H2. As dimensões das carreiras contemporâneas e de desenhos do trabalho se relacionarão positivamente e serão preditoras dos desfechos de engajamento;

H3. As dimensões das carreiras contemporâneas e de desenhos do trabalho se relacionarão negativamente e serão preditoras dos sintomas de adoecimento mental (ansiedade, depressão e estresse).

Com vistas a compreender melhor o cenário de mudanças amplamente descrito pela literatura científica (Baruch, et al., 2015; Baruch \& Vardi, 2016), optou-se, neste estudo, por investigar a transformação em empresas do ramo da Tecnologia de Informação (TI). Este segmento tem sido associado às carreiras contemporâneas e pós-corporativas, uma vez que: (a) os vínculos ilustram novos formatos de contratação (Softex, 2012); (b) os times operam de forma descentralizada e (c)
contemplar de igual forma diferentes aspectos de suas vidas, como os de mãe e trabalhadora 作 (Schaufeli, 2012).; (3) os formatos alternativos de carreira, como 
atuam em uma dinâmica na qual o comprador tem certa independência do fornecedor, portanto maior fragilidade no vínculo - terceirizações e contratações de consultores (Softex, 2013).

\section{Método}

\section{Participantes}

Participaram deste estudo 220 colaboradores de três empresas de TI da cidade de Porto Alegre, Rio Grande do Sul, Brasil. As empresas foram selecionadas por conveniência, e a pesquisa foi divulgada aos potenciais participantes conforme instruções fornecidas pelos responsáveis pela área de Recursos Humanos $(\mathrm{RH})$ que autorizaram a coleta de dados. A Empresa 01 possui 226 colaboradores e atua oferecendo espaços em seus servidores para armazenamento de conteúdo web. A Empresa 02 possui 200 funcionários e atua oferecendo diferentes soluções de TI, com foco em Design, inovação e software. A Empresa 03 possui em torno de 250 colaboradores e oferece consultoria em metodologia, ferramentas de apoio ao desenvolvimento de software e desenvolvimento e manutenção de aplicativos. Os profissionais que compuseram a amostra eram, em sua maioria, responsáveis por oferecer suporte especializado em diferentes etapas do processo de desenvolvimento e sustentação de software $(68 \%)$. As atividades por eles realizadas envolviam planejar, implementar, dar suporte e monitorar a infraestrutura de tecnologia, bem como desenvolver e programar softwares. Uma parcela menor de profissionais da amostra (31,37\%) atuava nas áreas de apoio administrativo das empresas. Dentre os participantes que concluíram a pesquisa, 38 eram colaboradores da Empresa 01, 97 da Empresa 02, e 124 da Empresa 03.

\section{Instrumentos}

Os participantes do estudo responderam um questionário online constituído por perguntas para aferir dados sociodemográficos (idade, sexo, nível educacional, carga horária de trabalho, setor da organização, tempo de empresa e número de empregos anteriores) e escalas para avaliar atitudes em relação à carreira, Desenho do Trabalho (DT), Engajamento no Trabalho (ET) e Ansiedade, Depressão e Estresse (ADE). As variáveis e instrumentos empregados para análise encontram-se descritos a seguir:

Atitudes em relação à carreira. Aplicou-se três escalas com esta finalidade. Uma delas foi a Escala de Atitudes de Carreira Sem-fronteiras (EACSF), adaptada por Oliveira, Zanon, Silva, Pinhatti, Gomes \& Gauer (2010) para a amostra. A escala é composta por 10 afirmativas que devem ser respondidas em escala Likert de cinco pontos. A medida contempla quatro itens de Mobilidade Física (MF) que expressam o desejo de se movimentar frequentemente entre empregadores independentes (alpha=0,79) e seis itens de Mobilidade Psicológica (MP), avaliando as preferências por mobilidade no contexto de trabalho e confiança de que a carreira pessoal pode se dar de forma independente ao empregador $(a l p h a=0,87)$. Aplicou-se também a Escala de Atitudes de Carreira Proteanas (EACP), adaptada para o Brasil por Oliveira, Zanon, Silva, Pinhatti, Gomes e Gauer (2009). Trata-se de um instrumento com 11 itens, avaliados por meio de uma escala Likert de cinco pontos. A medida é composta seis itens que avaliam Autogerenciamento (AG), que representa a responsabilidade e o controle que a pessoa possui sobre a própria carreira $(a \not p h a=0,78)$ e Direcionamento para Valores (DV), representando a priorização dos valores pessoais nas tomadas de decisão de carreira e na avaliação do que é sucesso $(a \not p h a=0,71)$. Por fim, aplicou-se a Escala de Parâmetros do Modelo de Carreira Caleidoscópica (EPMCC) (Bandeira, Andrade, Nora, Braga, \& Oliveira, 2019). O instrumen- to de 19 itens tem o intuito de avaliar os níveis de Autenticidade (A), que representa o direcionamento para valores e busca por atividades significativas ou ligadas a motivação pessoal $(\alpha=0,76)$; Balanço (B) que demonstra a busca por equilíbrio entre diferentes papéis de vida $(\alpha=0,81)$; e Crescimento (C) com foco na necessidade de um trabalho estimulante, busca por aprendizado, avanço e realização profissional $(\alpha=0,84)$. As assertivas apresentadas devem ser respondidas por meio de uma escala Likert de 5 pontos.

Características do trabalho. Utilizou-se o Questionário de Desenho no Trabalho (QDT) versão brasileira da Work Design Questionnaire (WDQ). O instrumento original foi proposto por Morgeson e Humphrey (2006) com a finalidade de avaliar características do trabalho de modo compreensivo e multidisciplinar. Trata-se de uma medida composta por quatro dimensões e 18 subdimensões relacionadas a aspectos motivacionais do trabalho, que são: (1) Características da Tarefa (Autonomia na planificação do trabalho; Autonomia de decisão e realização; Variedade de tarefas; Significado da tarefa; Identificação da tarefa; Feedback do trabalho), (2) Características de Conhecimento (Complexidade do trabalho; Processamento de informação; Solução de problemas; Especialização), (3) Características Sociais (Suporte Social; Interdependência; Interações fora da organização; Feedback dos outros) e (4) Características Contextuais (Ergonomia; Demandas Físicas; Condições de trabalho; Uso de equipamentos). O instrumento original apresentou índice de confiabilidade médio de 0,87 para todas subescalas (apenas uma subescala da dimensão Características Contextuais, a qual avalia aspectos ergonômicos do trabalho, apresentou alpha de Cronbach inferior a 0,70) (Morgeson \& Humphrey, 2006). Dois estudos foram conduzidos para obter evidências de validade do instrumento para o Brasil. O primeiro, uma análise fatorial exploratória da estrutura do modelo, e o segundo, a confirmação do ajuste da estrutura encontrada e da robustez da taxonomia proposta. Indicou-se uma solução composta por 18 subcategorias e 71 itens, que apresenta índices psicométricos considerados adequados (alpha igual ou superior a 0,70 para todos os fatores) (Borges-Andrade et al., 2019). Optou-se por utilizar o modelo de quatro fatores nas análises de Regressão e de Rede, uma vez que, teoricamente, esta solução também se mostra adequada para uso.

Engajamento no Trabalho (ET). Foi utilizada a Escala Utrecht de Engajamento no Trabalho (UWES) de nove itens. A UWES-9 é o instrumento mais utilizado, internacionalmente, para avaliar o Engajamento no Trabalho (ET) e no Brasil apresentou índice de confiabilidade adequado $(\alpha=$ 0,95). Trata-se de um instrumento com pontuação categorizada em escala Likert de sete pontos. Para fins de análise dos resultados, utilizou-se o escore geral de ET, seguindo a orientação de Vazquez, Pacico, Magnan, Hutz e Schaufeli (2016).

Sintomas de Ansiedade, Depressão e Estresse. Para avaliar estados emocionais negativos, optou-se pela utilização da Escala de Ansiedade, Depressão e Estresse de 21 itens (EADS-21). Tal ferramenta foi adaptada e apresentou evidências de validade para população brasileira adulta. Trata-se de um instrumento respondido em escala Likert de quatro pontos ( $\alpha=0,92$ para a escala de depressão, $\alpha=0,90$ para a de ansiedade e $\alpha=0,86$ para a de estresse) (Vignola $\&$ Tucci, 2014).

\section{Procedimentos de Coleta e Cuidados Éticos}

Para a seleção das instituições, identificou-se, por meio da rede LinkedIn, empresas com foco em desenvolvimento e licenciamento de software customizável ou não, ou em formato de consultoria, suporte técnico, ou manutenção e outros serviços em TI. No total, 31 empresas foram abordadas via e-mail disponibilizado no site ou através do próprio LinkedIn do profissional responsável 
pela área de Recursos Humanos (RH). Das empresas acessadas, 23 não retornaram o convite, cinco se interessaram pela proposta, mas não aceitaram participar da pesquisa e três foram incluídas no estudo. Estas disponibilizaram um questionário on-line para os seus colaboradores, divulgado por meio de canais de comunicação internos das empresas, porém hospedado na conta das pesquisadoras responsáveis e com acesso restrito na plataforma Qualtrics.

Antes de iniciar a aplicação do questionário, foi solicitado e recebido o consentimento dos locais onde a pesquisa ocorreu e os documentos foram submetidos ao Comitê de Ética da Universidade. Após este procedimento, estabeleceu-se o período de aplicação da pesquisa, de três semanas em cada local, contemplando os meses de setembro e outubro de 2018. Todos os colaboradores com mais de 18 anos, sediados em Porto Alegre, receberam informações sobre o estudo e assinaram o Termo de Consentimento Livre e Esclarecido (TCLE) para então responderem ao questionário.

\section{Procedimentos de Análise de Dados}

Os resultados foram analisados considerando as subdimensões dos constructos Carreira Proteana (Direcionamento para Valores e Autogerenciamento), Carreira Sem-Fronteira (Mobilidade Física e Mobilidade Psicológica) e Parâmetros de Carreira Caleidoscópica (Autenticidade, Balanço e Crescimento). Quanto ao Questionário de Desenho do Trabalho (QDT), utilizou-se as quatro dimensões: Características da Tarefa, do Conhecimento, Sociais e Contextuais. Quanto às medidas relacionadas à saú- de, utilizou-se o modelo unifatorial de Engajamento no Trabalho e foram unificadas as medidas de Ansiedade, Depressão e Estresse. $\mathrm{O}$ argumento psicométrico utilizado para tal unificação baseia-se na tentativa de diminuir o efeito de base - floor effect (Bech, Olsen, Kjoller, \& Rasmussen, 2003). Em amostras não clínicas, há uma tendência de os respondentes obterem escores baixos, levando a uma alta associação entre os fatores.

Partindo-se do pressuposto que o modelo estudado é um sistema complexo, utilizou-se um método exploratório com base na teoria dos grafos, a Análise de Redes, para identificar e descrever os padrões de relação entre variáveis (Machado, Vissoci, \& Epskamp, 2015). Para tanto, foi utilizado o software livre R, por meio do qual codificou-se as relações condicionais, nodewise L1-regularized, com vistas a controlar o efeito de variáveis aleatórias (Borsboom \& Cramer, 2013). Com o objetivo de identificar quais variáveis predizem níveis de Engajamento no Trabalho e Sintomas de Ansiedade, Depressão e Estresse, utilizou-se a Análise de Regressão Hierárquica (método stepwise). Optou-se pelo método de regressão stepwise uma vez que a associação entre as variáveis aqui propostas é pouco investigada na literatura, tratando-se, portanto, de um estudo exploratório (Abbad \& Torres, 2002). A referida técnica de aprendizado de máquina permite a seleção dos preditores mais importantes sem a interferência do pesquisador, tomando a medida da variância explicada (e seu incremento) como critério de inclusão de variáveis no modelo final. Foram verificados os pressupostos de normalidade, homocedasticidade e esfericidade para os dados da amostra. Os três pressupostos fo-

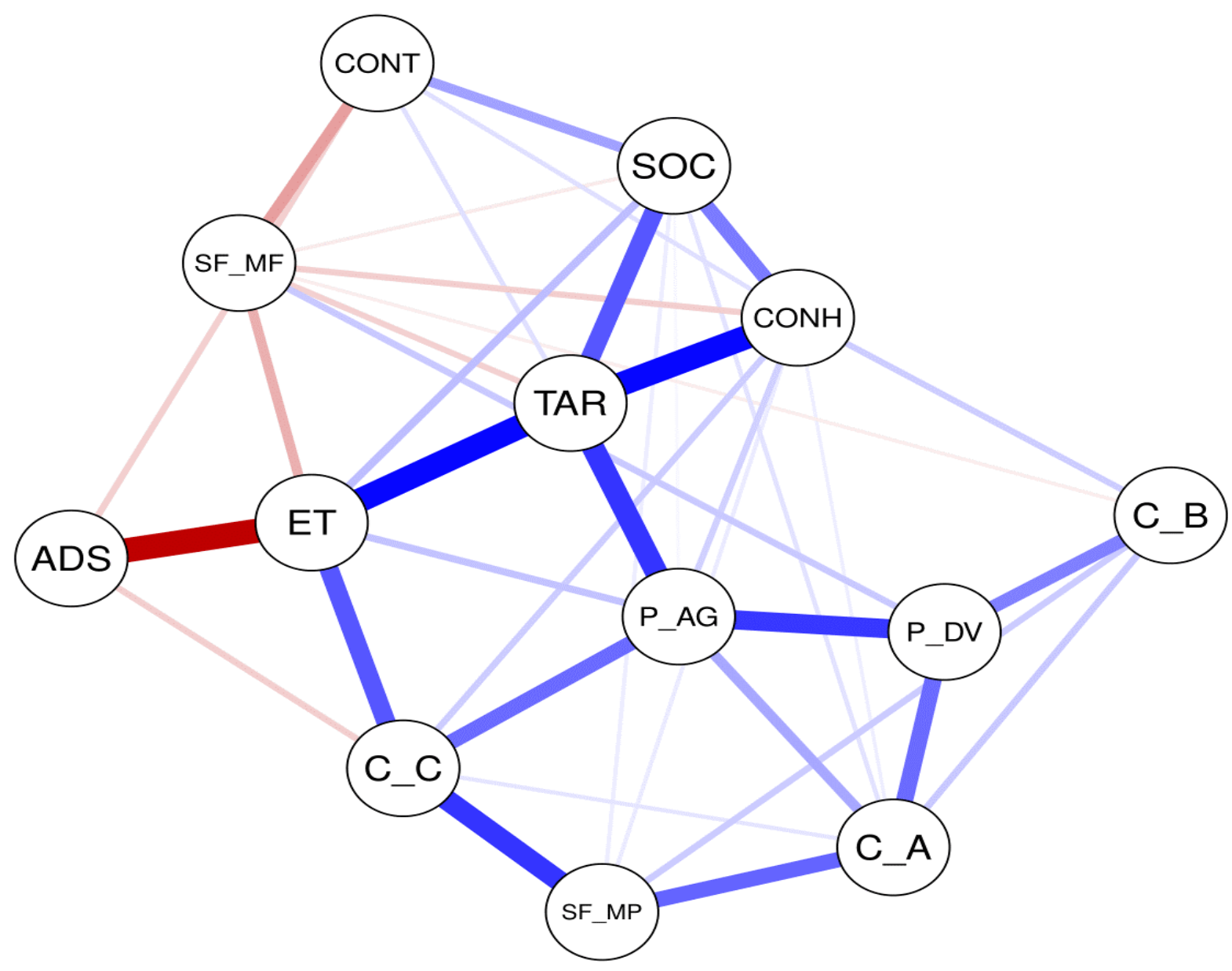

Figura 1. Rede de correlações parciais entre as variáveis investigadas.

Legenda: SF_MP: Mobilidade Psicológica; SF_MF: Mobilidade Física; C_A: Autenticidade; C_B: Balanço; C_C: Crescimento; P_AG: Autogerenciamento; P_DV: Direcionamento para Valores; TAR: Características da Tarefa; CONH: Características do Conhecimento; SOC: Características Sociais; CONT: Características de Contexto; ET: Engajamento no Trabalho; EADS: Estresse, Ansiedade e Depressão. Esta figura apresenta o padrão de relação entre todas as variáveis do sistema. 
ram atendidos em todas as dimensões avaliadas. Como ferramenta para obter tais análises, utilizou-se o Software estatístico SPSS.

\section{Resultados}

A amostra final do estudo foi composta por 220 participantes, dos quais, $72,3 \%$ são homens, $64,4 \%$ solteiros, $41,1 \%$ cursam e $27,7 \%$ já concluíram o ensino superior. Esta composição parece ser ilustrativa da população que atua na área de TI no Brasil.

A Análise de Redes buscou identificar o padrão de relação entre todas as variáveis do sistema. Na Figura 1, está representada a rede de correlações parciais das variáveis investigadas. $\mathrm{O}$ resultado obtido por meio desta análise é um objeto gráfico bidimensional, em que as variáveis são representadas por vértices (círculos) e a relação entre as variáveis por arestas (linhas). A intensidade das arestas indica a magnitude das relações e as cores indicam sua direção (azuis são positivas, vermelhas são negativas) (Machado et al., 2015).

Tabela 1

Coeficientes de regressão dos preditores de Engajamento no Trabalho pelo modelo stepwise

\begin{tabular}{cccc}
\hline Preditores & B & Beta & $p$ \\
\hline TAR & 0,78 & 0,43 & $\leq, 001$ \\
C_C & 0,53 & 0,30 & $\leq, 001$ \\
SF_MF & $-0,23$ & $-0,18$ & $\leq 0,01$ \\
C_B & $-0,17$ & $-0,12$ & 0,02 \\
\hline
\end{tabular}

Nota. B = coeficiente de regressão não padronizado; Beta = coeficiente de regressão padronizado; $\mathrm{p}$ = nível de significância estatística. Legenda: SF_MF: Mobilidade Física; C_B: Balanço; C_C: Crescimento; TAR: Características da Tarefa.

Destaca-se que a associação das medidas Característica da Tarefa e Crescimento com Engajamento no Trabalho foi fraca, embora positiva (correlação parcial de $r=0,29$ e $r=0,20$, respectivamente). Identifica-se ainda uma associação positiva, porém de muito pequena magnitude entre Autogerenciamento e Características Sociais com este desfecho (correlação parcial de $r=0,07$ e r $=0,08)$. Já Mobilidade Física e Engajamento no Trabalho tiveram uma associação de pequena magnitude, porém negativa $(r=-0,09)$. Também foi possível observar a relação negativa e fraca estabelecida entre as duas variáveis de desfecho deste estudo, Engajamento no Trabalho e Ansiedade, Depressão e Estresse $(r=-0,30)$. Por

Tabela 2

Coeficientes de regressão dos preditores de Engajamento no Trabalho pelo modelo stepwise

\begin{tabular}{cccc}
\hline Preditores & B & Beta & \multicolumn{1}{c}{ B } \\
\hline C_C & $-0,34$ & $-0,26$ & $\leq, 01$ \\
CONT & $-0,13$ & $-0,19$ & $\leq, 01$ \\
CON & 0,13 & 0,26 & $-0,01$ \\
TAR & $-0,48$ & $-0,17$ & 0,03 \\
\hline
\end{tabular}

Nota. B = coeficiente de regressão não padronizado; Beta = coeficiente de regressão padronizado; p = nível de significância estatística. Legenda: C_C: Crescimento; CONT: Características do contexto; CON: Características do conhecimento; TAR: Características da Tarefa; fim, merece destaque o fato de que as dimensões das diferentes expressões das carreiras contemporâneas (proteana, sem-fronteiras e caleidoscópica) investigadas não se mostraram diretamente relacionadas, à exceção das dimensões de autogerenciamento (P_AG) e direcionamento para valores (P_DV) da carreira proteana. Além disso, todas as dimensões dos diferentes estilos de carreira (à exceção de mobilidade física e balanço) estiveram diretamente relacionadas ao autogerenciamento, variável que remete à autonomia nas tomadas de decisão sobre a forma de conduzir a própria carreira.

Para identificar o poder de predição das variáveis independentes em relação ao Engajamento no Trabalho e à Ansiedade, à Depressão e ao Estresse, foram realizadas duas análises de Regressão Linear pelo método stepwise. A primeira delas incluiu as variáveis independentes Mobilidade Física, Mobilidade Psicológica, Direcionamento para Valores, Autogerenciamento, Autenticidade, Balanço e Crescimento (Atitudes de Carreira), Características da
Tarefa, do Conhecimento, Sociais e do Contexto (Desenho do Trabalho) e, como variável dependente, o Engajamento no Trabalho. Identificou um modelo com quatro preditores de Engajamento no Trabalho $[F(4,215)=41,38, p \leq 0,001]$, explicando cerca de $43 \%$ de sua variância. Os coeficientes não padronizados e padronizados de regressão dos preditores são apresentados na Tabela 1.

Destaca-se, a partir dos resultados obtidos na Análise de Regressão, o fato de três dos quatro fatores que predizem Engajamento no Trabalho corresponderem a dimensões das Carreiras Contemporâneas. Dentre todos os fatores que predizem Engajamento no Trabalho, dois são considerados promotores e dois supressores do desfecho. Na amostra investigada, ter um trabalho estimulante, que apresente desafios e promova o desenvolvimento, contribui para aumentar os índices de Engajamento no Trabalho. Entretanto, buscar o equilíbrio entre o papel do trabalho e outros papéis da vida tem efeito contrário, contribuindo para diminuir o engajamento das pessoas. Ainda em relação aos fato- res de carreira, o fator Mobilidade Física da Escala de Atitude de Carreira Sem-Fronteiras também tem efeito supressor do Engajamento no Trabalho. Em relação à percepção das características de trabalho, a variável Característica da Tarefa foi aquela que mais contribuiu com o Engajamento no Trabalho. Tal resultado indica que fatores relacionados à autonomia do profissional, significado e identidade do trabalho bem como feedback da própria atividade são os fatores que melhor explicam o Engajamento no Trabalho. Aspectos sociais, ergonômicos e do conhecimento não se mostraram significativos no modelo explicativo de Engajamento.

As mesmas Escalas de Carreira e de Desenho no Trabalho foram utilizadas para explicar os escores de Ansiedade, Depressão e Estresse. A Tabela 2 resume os achados desta regressão. As variáveis capazes de explicar as variações de Ansiedade, Depressão e Estresse foram Crescimento, Características do Contexto, do Conhecimento e da Tarefa. O modelo final apresentou índices de ajuste adequados $(F(4,215)=13, p \leq 0,01)$, explicando apenas $18 \%$ da variação nos indicadores de saúde mental dos participantes. Tal fato indica que apesar do aumento da complexidade no contexto de trabalho, Ansiedade, Depressão e Estresse podem ser explicados por outros fatores que não os escolhidos para análise neste estudo.

Em relação aos sintomas de Ansiedade, Depressão e Estresse, a Análise de Regressão permitiu identificar que apenas uma variável de carreira, o fator Crescimento, contou para explicação do desfecho. A relação estabelecida foi negativa, portanto, em empresas nas quais mais oportunidades de desenvolvimento e crescimento são apresentadas, menos os profissionais apresentarão sintomas pre- 
judiciais a sua saúde. Os resultados da Regressão ainda indicaram que poder realizar o trabalho com autonomia e perceber que ele tem sentido ajuda a reduzir os sintomas de Ansiedade, Depressão e Estresse. Da mesma forma contar com as condições ergonômicas adequadas contribui para a diminuição dos sintomas negativos. De forma contrária, a complexidade nas informações necessárias para resolver os problemas no trabalho podem intensificar os sintomas.

\section{Discussão}

O objetivo deste estudo foi compreender como se estabelece a relação entre as características do Desenho de Trabalho e as atitudes e comportamentos de carreira em contextos de trabalho de profissionais de TI. Objetivou-se, ainda, identificar os desfechos observados em medidas associadas à saúde do trabalhador. Ao abordar uma aproximação dos campos da Psicologia Organizacional e da Orientação Profissional, avanços foram apresentados.

Como em estudos anteriores com amostras diversas (Oliveira \& Gomes, 2014), os resultados desta pesquisa indicaram que os profissionais de TI acessados apresentam comportamentos típicos das carreiras contemporâneas, ou seja, são ativos e autônomos na gestão de suas carreiras. Em relação aos principais achados, as análises de rede confirmaram parcialmente a H1. Embora todas as dimensões de carreira (menos mobilidade física e balanço) tenham tido relação direta com o autogerenciamento de carreira, reforçando a ideia de que este é um elemento central das diferentes expressões de carreira contemporâneas (Baruch et al., 2015; Oliveira et al., 2009), nem todas as dimensões analisadas tiveram relação entre si.

A segunda hipótese deste estudo se mostrou parcialmente correta, uma vez que as atitudes e comportamentos em relação à carreira contribuíram mais que o desenho do trabalho para explicar a variação nos níveis de engajamento no trabalho. Além disso, evidenciaram que a única dimensão do desenho do trabalho que ajudou a explicar a variância do engajamento foi a que diz respeito à autonomia para a realização das tarefas e ao sentido que é atribuído a elas pelo trabalhador. Tal fator, juntamente com a busca de desafios e crescimento dentro da organização, pode, segundo a literatura, resultar em colaboradores mais satisfeitos e engajados no trabalho (Berg, Dutton, \& Wrzesniewski, 2008). Em contrapartida, a busca por balanço entre os diferentes papeis ocupados pelo trabalhador, os quais demandariam maior tempo e dedicação a atividades que não estão relacionadas ao trabalho (Greenhaus \& Kossek, 2014) contribuiriam para explicar variações negativas no engajamento. Assim também se dá a relação entre o interesse e a disposição para se movimentar entre setores e projetos e o Engajamento no Trabalho. Destaca-se ainda o fato de que alguns dos aspectos subjetivos de carreira, como Direcionamento para Valores, Mobilidade Psicológica e Autenticidade, não foram evidentes na promoção de desfechos positivos para o contexto organizacional. Apesar dos comportamentos e atitudes de carreira contemporâneos serem evidentes na amostra, nas organizações investigadas os fatores de carreira relacionados a formação da identidade de trabalho dos profissionais não parecem explicar variações em engajamento.

Se, por um lado, a literatura indica que pessoas engajadas também apresentam níveis de saúde mais elevados (Schaufeli $\&$ Taris, 2014), por outro tem-se apontado que os novos comportamentos de carreira podem estar promovendo estados de adoecimento (Baruch \& Vardi, 2016). Neste sentido, os resultados das análises de regressão indicaram que dentro das empresas aqui investigadas, critérios relacionados à transformação do ambiente de trabalho e das carreiras não estão influenciando de forma expressiva as variações de estados de Ansiedade, Depressão e Estresse (18\%), apesar de ser significativo o seu efeito. Pode-se observar que a hipótese três foi parcialmente aten- dida e cabe destacar que três elementos do desenho do trabalho contribuíram para o resultado observado e apenas um elemento das atitudes e comportamentos de carreira contemporâneos.

Tomados em conjunto, entende-se que as atitudes da carreira dos profissionais contemporâneos, bem como as características dos desenhos do trabalho se relacionam e impactam nos talentos da área de TI. Mais especificamente, este estudo sugere ser fator central na promoção do Engajamento do Trabalho e na prevenção da Ansiedade, Depressão e Estresse as configurações relacionadas as Características da Tarefa, bem como a percepção de crescimento na carreira. Ademais, estímulos relacionados as características do conhecimento ou mesmo as configurações de contexto podem prejudicar o desenvolvimento saudável do trabalho. O presente estudo convida a refletir sobre a necessidade de considerar novas configurações dos desenhos do trabalho na composição do contexto de emprego para trabalhadores que tem a autonomia como característica marcante de carreira.

\section{Considerações Finais}

Aponta-se a necessidade de que os profissionais da área da Psicologia Organizacional e do Trabalho levem em consideração que a maneira como as pessoas se relacionam com o trabalho tem mudado. Da mesma forma, profissionais da área de Orientação Profissional e de Carreira podem se beneficiar da compreensão da dinâmica dos contextos de trabalho para apoiar e facilitar a promoção das escolhas e desenvolvimento das trajetórias de carreira dos seus orientandos. Uma compreensão teórica inclusiva, que leve em conta variáveis individuais e do contexto das organizações poderá potencializar práticas de gestão de pessoas mais adequadas para os desafios das organizações na contemporaneidade.

Orienta-se que ao propor novas configurações para o contexto de trabalho, práticas que valorizem a reflexão sobre a formação da identidade de carreira estejam incluídas. Um trabalho de educação para a carreira, com vistas a fortalecer o papel do direcionamento subjetivo de sucesso profissional pode ser uma alternativa para superar os modelos atualmente conhecidos. Orienta-se assim, que novas investigações ajudem a esclarecer como a construção do sentido e da identidade profissional pode ter espaço no contexto organizacional.

\section{Referências}

Abbad, G., \& Torres, C. V. (2002). Regressão múltipla stepwise e hierárquica em Psicologia Organizacional: aplicações, problemas e soluções. Estudos de Psicologia (Natal), 7(SPE), 19-29. https://doi.org/10.1590/S1413294X2002000300004

Bandeira, P. B., Andrade, A. L. De, Nora, M. de C. V., Braga, M. M., \& Oliveira, M. Z. de. (2019). Adaptação e Evidências de Validade da Escala de Parâmetros da Carreira Caleidoscópica. Revista Brasileira de Orientação Profissional, 20(1), 103-114. https://doi.org/10.26707/1984-7270/2019v20n1p103

Baruch, Y., Szűcs, N., \& Gunz, H. (2015). Career studies in search of theory: The rise and rise of concepts. Career Development International, 20(1), 3-20. https://doi.org/10.1108/cdi-11-2013-0137

Baruch, Y., \& Vardi, Y. (2016). A fresh look at the dark side of contemporary careers: Toward a realistic discourse. British Journal of Management, 27(2), 355372. https://doi.org/10.1111/1467-8551.12107

Bech, P., Olsen, L. R., Kjoller, M., \& Rasmussen, N. K. (2003). Measuring well-being rather than the absence of distress symptoms: a comparison of the SF-36 Mental Health subscale and the WHO-Five well-being scale. International journal of methods in psychiatric research, 12(2), 85-91. https://doi. org $/ 10.1002 / \mathrm{mpr} .145$

Berg, J. M., Dutton, J. E., \& Wrzesniewski, A. (2008). What is job crafting and why does it matter. Retrieved form the website of Positive Organizational Scholarship on April, 15, 2011. Recuperado em 10 de fevereiro de 2020, de https://positiveorgs.bus.umich.edu/wp-content/uploads/What-is-JobCrafting-and-Why-Does-it-Matter1.pdf 
Borges-Andrade, J. E., Peixoto, A. L. A., Queiroga, F., \& Pérez-Nebra, A. R. (2019). Adaptation of the Work Design Questionnaire to Brazil. Revista Psicologia Organizações e Trabalho, 19(3), 720-731. https://doi.org/10.17652/ rpot/2019.3.16837

Borsboom, D., \& Cramer, A. O. (2013). Network analysis: an integrative approach to the structure of psychopathology. Annual review of clinical psychology, 9, 91121. https://doi.org/10.1146/annurev-clinpsy-050212-185608

Cummings, T. G. (1978). Self-regulating work groups: A socio-technical synthesis. Academy of Management Review, 3(3), 625-634. https://doi. org/10.2307/257551

Dalanhol, N. S., Freitas, C. P. P., Machado, W. L., Hutz, C. S., \& Vazquez, A. C. S. (2017). Engajamento no trabalho, saúde mental e personalidade em oficiais de justiça. Revista Psico, 48(2), 109-119. https://doi.org/10.15448/1980$\underline{8623.2017 .2 .25885}$

Greenhaus, J. H., \& Kossek, E. E. (2014). The contemporary career: A work-home perspective. The Annual Review of Organizational Psychology and Organizational Behavior, 14(1), 361-388. https://doi.org/10.1146/annurev-orgpsych-031413-091324

Greenhaus, J. H., \& Powell, G. N. (2006). When work and family are allies: A theory of work-family enrichment. Academy of management review, 31(1), 7292. https://doi.org/10.5465/amr.2006.19379625

Hakanen, J. J., Schaufeli, W. B., \& Ahola, K. (2008). The Job DemandsResources model: A three-year cross-lagged study of burnout, depression, commitment, and work engagement. Work \& Stress, 22(3), 224-241. https:// doi.org/10.1080/02678370802379432

Hall, D. T., \& Las Heras, M. (2009). Long live the organisational career. In Vocational Psychological and Organisational Perspectives on Career, 3 (pp.181-196). Leiden, The Netherlands: Brill Sense. https://doi. org/10.1163/9789087909178 012

Hallberg, U. E., Schaufeli, W. B. (2006). "Same same" but different? Can work engagement be discriminated from job involvement and organizational commitment? European psychologist, 11(2), 119-127. https://doi. org/10.1027/1016-9040.11.2.119.

Harden, G., Boakye, K. G., \& Ryan, S. (2018). Turnover intention of technology professionals: A social exchange theory perspective. Journal of Computer Information Systems, 58(4), 291-300. https://doi.org/10.1080/08874417.201 $\underline{6.1236356}$

Herrmann, A., Hirschi, A., \& Baruch, Y. (2015). The protean career orientation as predictor of career outcomes: Evaluation of incremental validity and mediation effects. Journal of Vocational Behavior, 88, 205-214. https://doi. org/10.1016/i.jvb.2015.03.008

Kašpárková, L., Vaculík, M., Procházka, J., \& Schaufeli, W. B. (2018). Why resilient workers perform better: The roles of job satisfaction and work engagement. Journal of Workplace Behavioral Health, 33(1), 43-62. https://doi. org/10.1080/15555240.2018.1441719

Kostal, J. W., \& Wiernik, B. M. (2017). A meta-analytic investigation of demographic differences in protean, boundaryless, and proactive career orientations. Career Development International, 22(5), 520-545. https://doi.org/10.1108/ cdi-08-2017-0139

Lassance, M. C., \& Sarriera, J. C. (2009). Carreira e saliência dos papéis: Integrando o desenvolvimento pessoal e profissional. Revista Brasileira de Orientação Profissional, 10(2), 15-31. Recuperado em 08 de fevereiro de 2020, de http://pepsic.bvsalud.org/scielo.php?script=sci arttext\&pi$\mathrm{d}=$ S1679-33902009000200004\&lng $=$ pt\&tlng $=\mathrm{pt}$

Machado, W. L., Vissoci, J., \& Epskamp, S. (2015). Análise de rede aplicada à Psicometria e à avaliação psicológica. In C. S. Hutz, D. R. Bandeira, \& C. M. Trentini (Eds.), Psicometria (pp. 125-146). Porto Alegre.

Mainiero, L. A., \& Sullivan, S. E. (2005). Kaleidoscope careers: An alternate explanation for the "opt-out "revolution. Academy of Management Perspectives, 19(1), 106-123. https://doi.org/10.5465/ame.2005.15841962

McDonald, P., Brown, K., \& Bradley, L. (2005). Have traditional career paths given way to protean ones? Evidence from senior managers in the Australian public sector. Career Development International, 10(2), 109-129. https://doi.org/10.1108/13620430510588310

Morgeson, F. P., \& Humphrey, S. E. (2006). The Work Design Questionnaire (WDQ): developing and validating a comprehensive measure for assessing job design and the nature of work. Journal of applied psychology, 91(6), 13211329. https://doi.org/10.1037/0021-9010.91.6.1321

Morgeson, F. P., Garza, A. S., \& Champion, M. A. (2013). Work design in handbook of psychology. In Industrial and Organizational Psychology, 12 (2 ed., pp. 525-559). https://doi.org/10.1002/0471264385.wei1217

Oldham, G. R., \& Fried, Y. (2016). Job design research and theory: Past, present and future. Organizational Behavior and Human Decision Processes, 136, 20-35. https://doi.org/10.1016/i.obhdp.2016.05.002

Oliveira, M. Z., \& Gomes, W. B. (2014). Estilos reflexivos e atitudes de carreira proteana e sem fronteiras nas organizações contemporâneas brasileiras. Revista Psicologia Organizações e Trabalho, 14(1), 105-118. Recuperado em 07 de fevereiro de 2020, de http://pepsic.bvsalud.org/scielo.php? script=sci arttext\&pid $=$ S1984-66572014000100009\&lng=pt\&tlng=pt.
Oliveira, M. Z., Zanon, C., Silva, I. S., Pinhatti, M. M., Gomes, W. B., \& Gauer, G. (2009). Avaliação do autogerenciamento e do direcionamento de carreira: estrutura fatorial da escala de atitudes de carreira proteana. Gerais : Revista Interinstitucional de Psicologia, 2(2), 160-169. Recuperado de http://pepsic. bvsalud.org/scielo.php?script=sci arttext\&pid=S1983-82202009000200011

Oliveira, M. Z., Zanon, C., Silva, I. S., Pinhatti, M. M., Gomes, W. B., \& Gauer, G. (2010). Validação da Versão Brasileira da Escala de Atitudes de Carreira Sem-Fronteiras. Arquivos Brasileiros de Psicologia, 62(3), 106-114. Recuperado em 8 de Fevereiro de 2020, em http://pepsic.bvsalud.org/scielo.php?scrip$\mathrm{t}=$ sci arttext\&pid $=$ S1809-52672010000300012

Parker, S. K., Morgeson, F. P., \& Johns, G. (2017). One hundred years of work design research: Looking back and looking forward. Journal of applied psychology, 102(3), 403. https://doi.org/10.1037/apl0000106

Peiperl, M., \& Baruch, Y. (1997). Back to square zero: The post-corporate career. Organizational dynamics, 25(4), 7-22. https://doi.org/10.1016/s00902616(97)90033-4

Ribeiro, M. A., \& Zerbini, T. (2012). Síntese das discussões e propostas do Grupo de Trabalho: Interfaces entre a orientação profissional, psicologia organizacional e do trabalho e administração. Revista Brasileira de Orientação Profissional, 13(1), 117-120. Recuperado em 8 de Fevereiro de 2020, em https://www. redalyc.org/pdf/2030/203023770013.pdf

Schaufeli, W. B. (2012). Work engagement: What do we know and where do we go? Romanian Journal of Applied Psychology, 14(1), 3-10. Recuperado em 08 de Fevereiro de 2020, de https://lirias.kuleuven.be/retrieve/307310

Schaufeli, W. B. (2017). Applying the job demands-resources model. Organizational Dynamics, 2(46), 120-132. https://doi.org/10.1016/j.orgdyn.2017.04.008

Schaufeli, W. B., \& Taris, T. W. (2014). A critical review of the Job DemandsResources Model: Implications for improving work and health. Bridging occupational, organizational and public health, 43-68. Springer, Dordrecht. https://doi.org/10.1007/978-94-007-5640-3 4

Softex, A. (2012). Indústria brasileira em perspectiva v2. Observatório Softex. Recuperado em 26 de Janeiro de 2020, de https://softex.br/download/ cadernos-tematicos-2012-observatorio-softex-industria-brasileira-softwareservicos-ti-em-perspectiva-versao-completa-portugues/

Softex , O. (2013). Mercado de trabalho e formação de mão de obra em TI. Cadernos Temáticos do Observatório. Observatório Softex, 114-118. Recuperado em 26 de Janeiro de 2020, de http://www.ftp.softex.br/Inteligencia/cadernos tematicos/cadernos tematico mercado de trabalho.pdf

Sullivan, S. E., \& Baruch, Y. (2009). Advances in career theory and research: A critical review and agenda for future exploration. Journal of management, 35(6), 1542-1571. https://doi.org/10.1177/0149206309350082

Vazquez, A. C., Pacico, J. C., Magnan, E., Hutz, C. S., \& Schaufeli, W. (2016). Avaliação do engajamento das pessoas com seu trabalho: a versão brasileira da escala Utrecht de Engajamento no Trabalho (UWES). In C. Hutz (Ed.), Avaliação em Psicologia Positiva (pp. 75-87). São Paulo: Hoegrefe.

Vignola, R. C. B., \& Tucci, A. M. (2014). Adaptation and validation of the depression, anxiety and stress scale (DASS) to Brazilian Portuguese. Journal of affective disorders, 155(2014), 104-109. https://doi.org/10.1016/i. jad.2013.10.031

Wiernik, B. M., \& Kostal, J. W. (2019). Protean and boundaryless career orientations: A critical review and meta-analysis. Journal of counseling psychology, 66(3), 280. https://doi.org/10.31234/osf.io/ftm2k

Zaza, I., \& Armstrong, D. J. (2017). Information Technology Professionals' Turnover Intentions: A Meta-Analysis of Perceived Organizational Factors. Recuperado em 08 de Fevereiro de 2020, de https://aisel.aisnet.org/icis2017/HumanBehavior/Presentations/2/ 